\title{
Solitaire AB stent-assisted coiling embolization for the treatment of ruptured very small intracranial aneurysms
}

\author{
JIFANG ZHANG $^{1,2}$, DONGHAI WANG ${ }^{1}$ and XINGANG LI ${ }^{1}$ \\ ${ }^{1}$ Department of Neurosurgery, Qilu Hospital, Shandong University, Jinan, Shandong 250012; \\ ${ }^{2}$ Department of Neurosurgery, Qingdao Municipal Hospital, Qingdao, Shandong 266071, P.R. China
}

Received July 1, 2014; Accepted March 16, 2015

DOI: $10.3892 /$ etm.2015.2826

\begin{abstract}
Recent advances in neuroradiological techniques have increasingly improved the diagnosis rate for very small aneurysms, particularly with the widespread use of three-dimensional cerebral angiography. However, the treatment of very small aneurysms remains a considerable challenge for neurosurgeons. Endovascular coiling has emerged as a potential treatment option for intracranial aneurysms. The aim of the present study was to evaluate the safety and efficacy of Solitaire AB stent-assisted coiling embolization for the treatment of ruptured very small intracranial aneurysms. This retrospective study included nine consecutive patients with ruptured very small intracranial aneurysms $(\leq 3 \mathrm{~mm})$ that underwent Solitaire AB stent-assisted coiling embolization. The aneurysms were located in the ophthalmic branch of the internal carotid artery $(n=2)$, the posterior communicating branch of the internal carotid artery $(n=4)$, the top of the basilar artery $(n=1)$ and the middle cerebral artery $(n=2)$. Solitaire AB stents were successfully implanted in all nine patients. Of the nice individuals, six patients exhibited complete occlusion at Raymond grade I and three patients exhibited occlusion at Raymond grade II. No aneurysm rupture was observed during the surgery. During the follow-up period of 8-13 months, no intracranial hemorrhage occurred. A total of seven patients underwent follow-up digital subtraction angiography at 5-10 months post-intervention. No recurrence of the aneurysms and no stenosis or occlusion of the parent arteries was observed. Therefore, Solitaire AB stent-assisted coil embolization was demonstrated to be a safe and effective treatment for ruptured very small intracranial aneurysms. The long-term efficacy of this technique may be improved by
\end{abstract}

Correspondence to: Dr Xingang Li, Department of Neurosurgery, Qilu Hospital, Shandong University, 107 Wenhua West Road, Jinan, Shandong 250012, P.R. China

E-mail:lixg@sdu.edu.cn

Key words: tiny intracranial aneurysm, Solitaire AB stent, endovascular treatment increasing the packing density around the aneurysmal neck and improving the hemodynamics.

\section{Introduction}

Very small intracranial aneurysms have been associated with intracranial hemorrhage following rupture. Although ruptured very small aneurysms can be treated by direct surgical clipping or endovascular embolization, these treatments are often highly complex and are associated with a high risk of complication (1). Currently, there is no consensus with regard to the definition of very small intracranial aneurysms; however, in general, aneurysms with a diameter of $\leq 3 \mathrm{~mm}$ are generally defined as very small (2). Recent advances in neuroradiological techniques, particularly the widespread use of three-dimensional (3D) cerebral angiography, have increasingly improved the diagnosis rate for very small aneurysms. However, the treatment of small aneurysms remains a considerable challenge for neurosurgeons (1).

Endovascular coiling has emerged as a potential treatment option for intracranial aneurysms. A number of studies have demonstrated that Solitaire AB stent-assisted coiling embolization is a safe and effective treatment for wide-necked or complex intracranial aneurysms (3-6). In addition, Zhao et al reported that Solitaire AB stents are effective for the treatment of very small $(\leq 3 \mathrm{~mm})$ and small $(3-10 \mathrm{~mm})$ ruptured and unruptured intracranial aneurysms (7). However, the use of Solitaire AB stents for the treatment of ruptured very small intracranial aneurysms has not been reported in the literature. The present retrospective study assessed nine patients with ruptured very small intracranial aneurysms that underwent Solitaire AB stent-assisted coiling embolization between July 2010 and December 2012. The aim of the current study was to investigate and evaluate the efficacy and safety of Solitaire AB stent-assisted coiling embolization for the treatment of ruptured very small intracranial aneurysms.

\section{Materials and methods}

Patients. The retrospective study was approved by the Ethics Committees of Qilu Hospital (Jinan, China) and Qingdao Municipal Hospital (Qingdao, China). Written informed consent was obtained from each subject or their representative. The subject population consisted of nine patients (male, 
7; female, 2) with ruptured very small intracranial aneurysms that underwent Solitaire AB stent-assisted coiling embolization at the Department of Neurosurgery at Qingdao Municipal Hospital between July 2010 and December 2012. The average

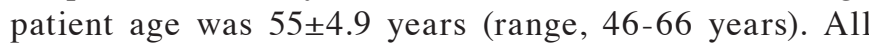
patients had a history of spontaneous subarachnoid hemorrhage (SAH). The study inclusion criteria were as follows: i) History of SAH confirmed by a head computed tomography examination, which showed the hemorrhage from the aneurysms; ii) very small aneurysms with a size of $\leq 3 \mathrm{~mm}$ in diameter diagnosed by digital subtraction angiography (DSA); iii) no other aneurysms in the brain; and iv) aneurysms meeting the indication for Solitaire AB stent-assisted coiling embolization (4-6). Exclusion criteria were as follows: i) No history of SAH; ii) intracranial hemorrhage not associated with the aneurysms; iii) intracranial hematoma with a volume of $\geq 30 \mathrm{ml}$; and iv) parent arteries that were tortuous or exhibited severe stenosis, which indicated that Solitaire AB stent-assisted coiling embolization was not suitable. A total of 10 aneurysms were identified among the nine patients, including eight patients with a single aneurysm and one patient with two aneurysms. The aneurysms were located in the ophthalmic branch of the internal carotid artery $(n=2$, including the case with two aneurysms), the posterior communicating branch of the internal carotid artery $(n=4)$, the top of the basilar artery $(n=1)$ and the middle cerebral artery $(n=2)$.

Procedure. All patients underwent systemic heparinization under general anesthesia $(500 \mathrm{mg} / \mathrm{ml}$ propofol and $200 \mu \mathrm{g} / 50 \mathrm{ml}$ dexmedetomidine). A $6 \mathrm{~F}$ arterial sheath (Terumo Holding Co. Ltd., Tokyo, Japan) was inserted following a puncture to the femoral artery using the Seldinger technique. Routine DSA with 3D reconstruction was performed for each patient to evaluate the aneurysm size and the proximal and distal diameters of the parent arteries, in order to clearly determine the anatomical association between the aneurysm and the parent artery and its branches (Figs. 1-3). Based on the DSA results, the appropriate surgical approach, operating angle, coil and stent were selected for each patient. A 6F ENVOY guiding catheter (Cordis Neurovascular, Inc., Miami Lakes, FL, USA) was inserted into the internal carotid artery at the level of the second cervical vertebra. A Rebar microcatheter (eV3 Endovascular, Inc., Plymouth, MN, USA) was conducted through a Traxcess guidewire (MicroVention, Inc., Tustin, CA, USA) and positioned in the parent artery past the aneurysmal neck. The Solitaire AB stent was advanced through the Rebar microcatheter and placed at the cephalic end of the microcatheter; however, the stent was not released. An Echelon 10 microcatheter (eV3 Endovascular, Inc.) with a very small 'L'-shaped tip was inserted into the aneurysmal sac or neck. Aneurysmal coiling was performed using an Axium 3D coil (eV3 Endovascular, Inc.) of appropriate diameter and length. After one or two coil loops were released to fill the aneurysmal cavity, the stent was slowly released, and the release was stopped after the microcatheter was fixed and the coil did not pulsate. The aneurysmal cavity was continuously filled with the coil until 1-2 cm of the coil remained inside the parent artery. The microcatheter was removed after the guidewire achieved stability. After the position of the coil was stabilized, the stent was completely released and the coil was isolated.

All patients received $300 \mathrm{mg}$ aspirin and $300 \mathrm{mg}$ Plavix (Sanofi Winthrop, Paris, France) orally or intragastrically prior to the intervention. Following the intervention, patients were treated with $100 \mathrm{mg}$ aspirin and $75 \mathrm{mg}$ Plavix per day. Plavix administration was terminated after 1 month, while aspirin treatment was stopped after 6 months. A lumbar puncture was performed daily, starting on the first day after the intervention, until no blood was observed in the cerebrospinal fluid. For patients with acute hydrocephalus, routine ventricular drainage was performed.

Postoperative evaluation and follow-up. The degree of occlusion was evaluated immediately post-procedure (Figs. 1-3). Occlusion degree was graded according to the modified Raymond scale (8) as follows: Grade I, complete occlusion (no contrast agents observed in the aneurysm); grade II, neck remnant (contrast agents observed in the aneurysmal neck); and grade III, partial occlusion (contrast agents observed in the aneurysmal cavity). All patients underwent follow-up via telephone and hospital visits at 1, 3, 6 and 12 months following the intervention. Patients underwent a follow-up DSA at $\sim 6$ months after the intervention (Figs. 1-3). Clinical outcome was evaluated according to the modified Rankin scale (mRS) (9): Grade 0, no symptoms; grade 1 , limited symptoms, but with no significant disability (able to perform daily duties and activities); grade 2, minor disability (unable to perform previous activities, but able to perform own bodily activities without assistance); grade 3, moderate disability (requiring limited assistance, but able to walk independently); grade 4, moderate to severe disability (unable to walk without assistance and unable to attend to own bodily activities without assistance); and grade 5, severe disability (bedridden, incontinent and requiring nursing care and attention).

\section{Results}

Surgical outcomes. The Hunt-Hess classification (10) of SAH was used to describe the severity of preoperative bleeding. Among the nine patients, 22.2\% ( $\mathrm{n}=2)$ were Hunt-Hess grade I, $22.2 \%(n=2)$ were grade II, $44.4 \%(n=4)$ were grade III and $11.1 \%(n=1)$ was grade IV. All patients underwent 3D-DSA immediately following the surgery, and the degree of occlusion was evaluated according to the Raymond scale. Among the nine patients, six patients exhibited complete occlusion at Raymond grade I and three patients exhibited occlusion at Raymond grade II.

No intraoperative hemorrhage due to aneurysm rupture was observed in any patient, and no acute thrombosis formed in the parent arteries. In addition, no problems were encountered in the deployment of stents and coils in any of the nine patients, and no operation-associated mortality or morbidity occurred.

Complications. Vasospasms occurred in one patient with an aneurysm in the middle cerebral artery (preoperative Hunt-Hess grade I). During surgery, $5 \mathrm{ml}$ Nimotop (Bayer Schering Pharma, Berlin, Germany) in $15 \mathrm{ml}$ saline was 
A

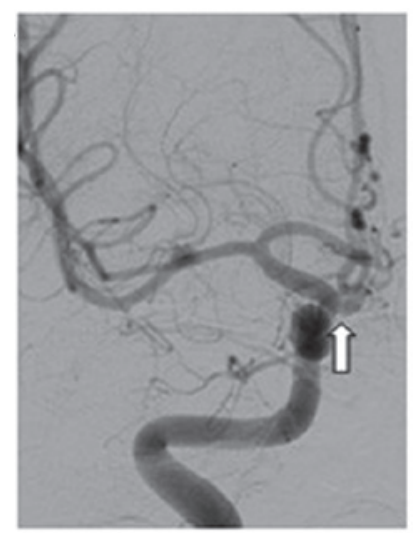

D

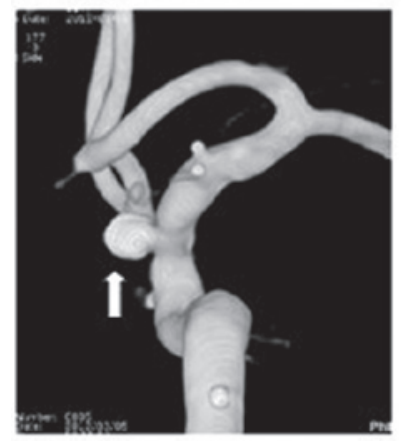

B

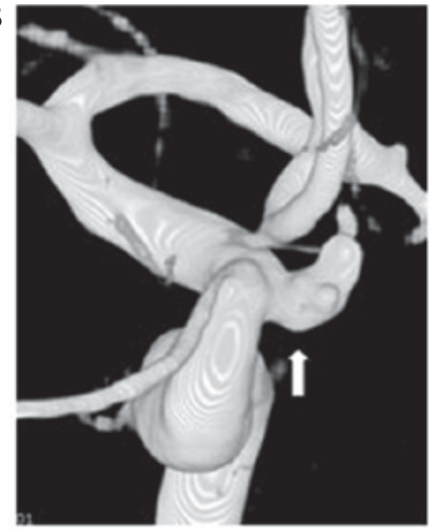

$\mathbf{E}$

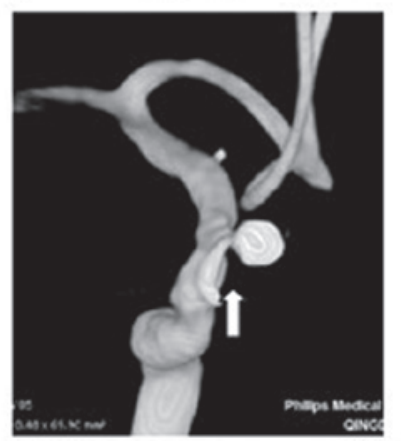

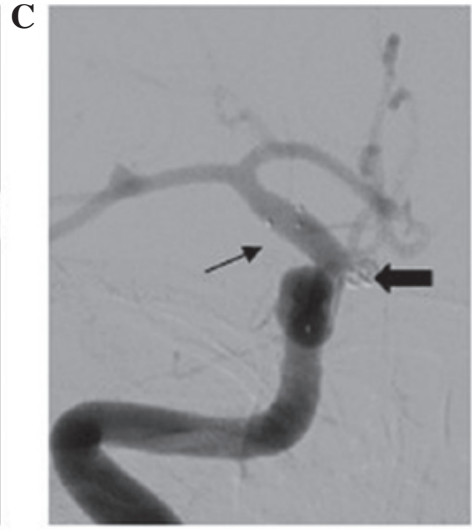

$\mathbf{F}$

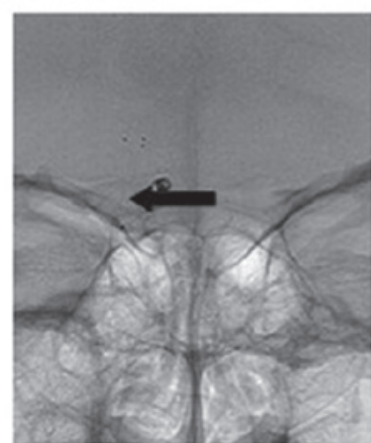

Figure 1. Digital subtraction angiography (DSA) images before and after Solitaire AB stent-assisted coiling embolization in a 49-year-old female that presented with a sudden headache and numbness in the left limb, and was hospitalized due to the onset of spontaneous subarachnoid hemorrhage. (A and B) DSA images show a very small aneurysm in the ophthalmic branch of the internal carotid artery (white arrow). (C) DSA image obtained immediately after Solitaire AB stent-assisted coiling embolization. The stent $(4 \times 20 \mathrm{~mm}$; narrow arrow) was placed in the parent artery past the aneurysmal neck, and the coil (Axium 3D; eV3 Endovascular, Inc., Plymouth, MN, USA; $0.2 \times 6 \mathrm{~cm}$; thick arrow) was under the stent in the parent artery. The degree of occlusion was Raymond grade II. (D-F) DSA images show dense coil packing (white arrows) in the aneurysm at 8 months after the intervention. The parent artery was patent without any stenosis, and the degree of occlusion was Raymond grade I. (F) Stent markers and the coil are indicated by the black arrow.

A

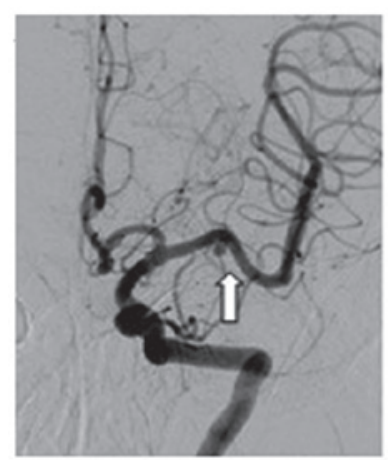

D

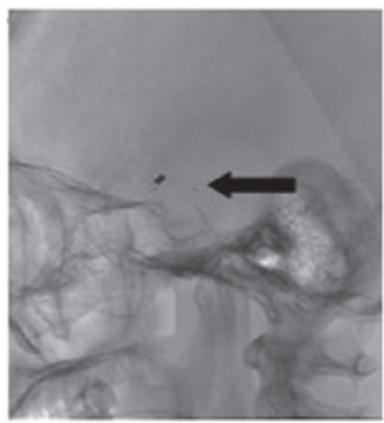

B

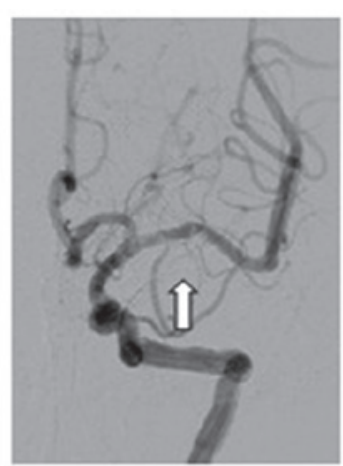

$\mathbf{E}$

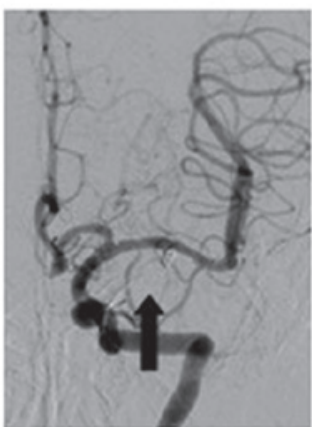

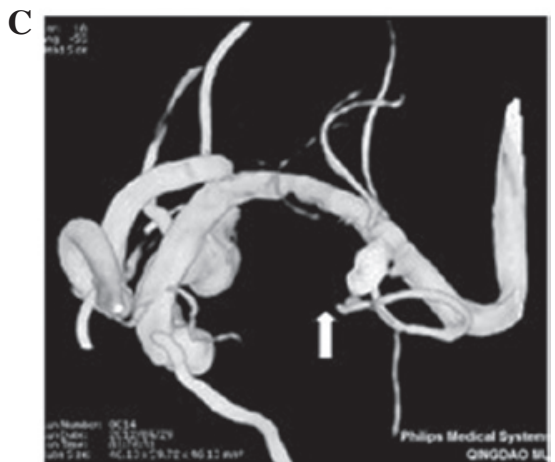

$\mathbf{F}$

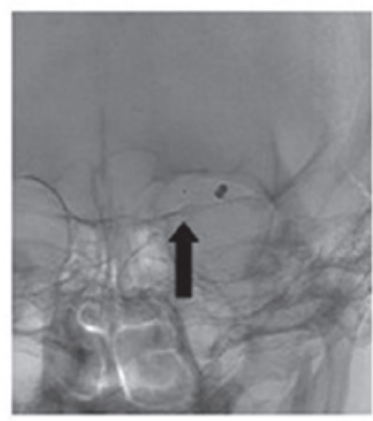

Figure 2. Digital subtraction angiography (DSA) images before and after Solitaire AB stent-assisted coiling embolization in a 60-year-old male that presented with a sudden headache, nausea and vomiting, and was hospitalized due to the onset of spontaneous subarachnoid hemorrhage. (A) DSA image shows a very small aneurysm (white arrow). (B and C) DSA images obtained immediately after Solitaire AB stent $(4 \times 15 \mathrm{~mm})$-assisted coiling embolization show dense coil packing (white arrow) and patent parent artery. The short tail of the coil (Axium 3D; eV3 Endovascular, Inc., Plymouth, MN, USA; 0.2x6 cm) remained in the parent artery and entered the aneurysm when the microcatheter was retrieved. The degree of occlusion was Raymond grade I. (D) Stent markers and the coil are indicated by the black arrow. (E and F) DSA images show dense coil packing (black arrows) in the aneurysm at 5 months after the intervention. The parent artery was patent without any stenosis. 


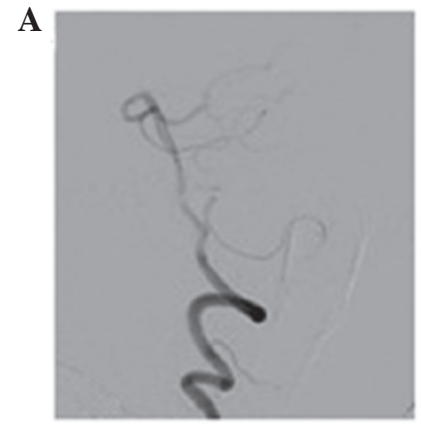

B

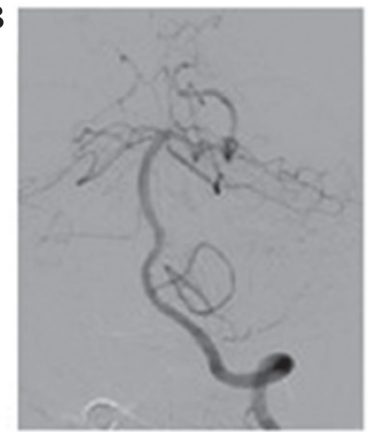

D

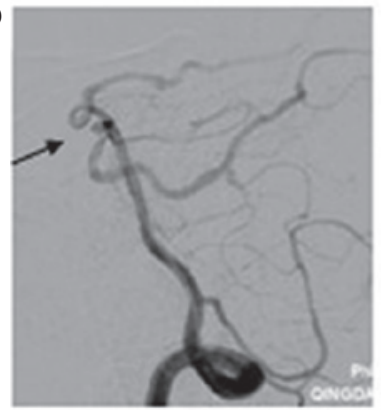

G

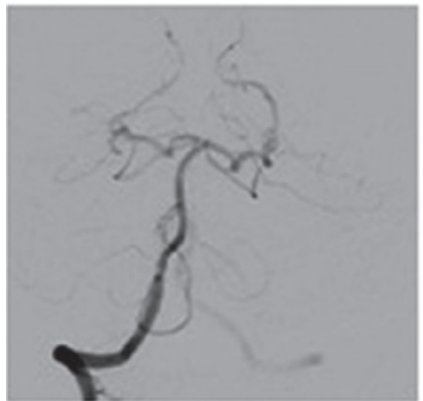

E

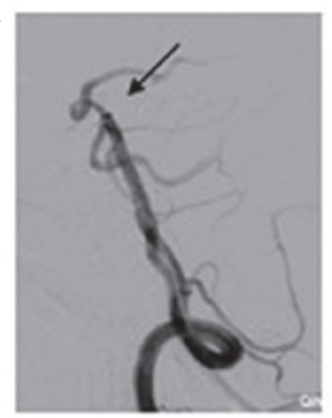

$\mathbf{H}$

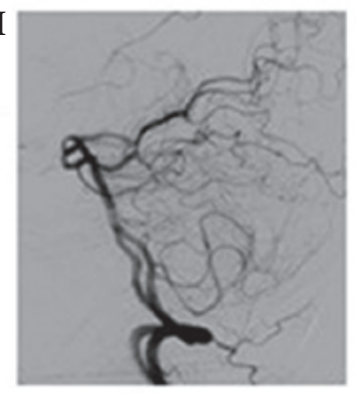

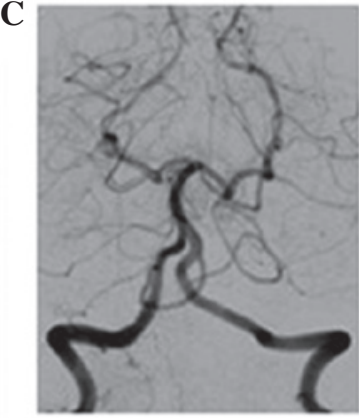

$\mathbf{F}$

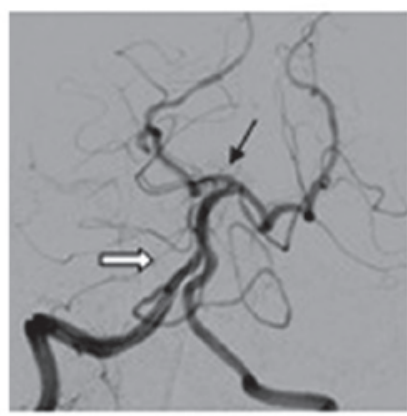

I

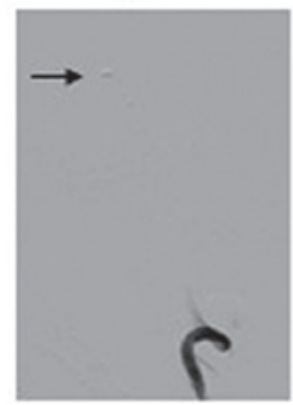

Figure 3. Digital subtraction angiography (DSA) images before and after Solitaire AB stent-assisted coiling embolization in a 66-year-old female who was transferred to the Qingdao Municipal Hospital (Qingdao, China) after suffering from a subarachnoid hemorrhage for 3 months. (A) Anterior and (B and C) lateral views from the DSA of the (A and B) left and (C) right vertebral arteries showing no abnormalities. (D) Three-dimensional-DSA image shows a very small aneurysm on the top of the basilar artery (black arrow). (E and F) DSA images obtained during Solitaire AB stent (4x15 mm)-assisted coiling embolization show the dense coil packing (black arrow) and patent parent artery. The degree of occlusion was Raymond grade I. (F) Stent markers and the coil are indicated by the white arrow. (G-I) DSA images obtained immediately after Solitaire AB stent-assisted coiling embolization show the patent parent artery. The degree of occlusion was Raymond grade I. (I) Stent markers and the coil are indicated by the black arrow.

administered via the guiding catheter at a rate of $5 \mathrm{ml} / 30 \mathrm{~min}$. DSA was conducted after each 5-ml infusion of Nimotop. Nimotop administration was ended if the vasospasm was relieved. No evident cerebral ischemia occurred postoperatively. All patients underwent a neurological examination, and no patients exhibited evident neurological dysfunction. A total of two patients with hydrocephalus underwent was drainage for a limited period ( $<7$ days), one patient with Hunt-Hess grade III and one patient was Hunt-Hess grade IV. One of the two patients (Hunt-Hess grade IV) subsequently underwent ventriculoperitoneal shunting.

Follow-up. All nine patients underwent follow-up for a period of 8-13 months (average, 10.6 months). A total of two patients (one patient with Hunt-Hess grade III and one patient with Hunt-Hess grade IV) exhibited mRS grade 2, while the other seven patients were classified as mRS grade 1 . Of the nine patients, seven patients underwent follow-up DSA at 5-10 months post-intervention. All seven of these patients exhibited complete occlusion at Raymond grade I. No recurrence or enlargement of the aneurysms occurred and no stenosis or occlusion were observed in the parent arteries.

\section{Discussion}

Very small aneurysms reportedly account for $\sim 7 \%$ of all ruptured aneurysms (11). However, very small aneurysms are rarely identified during clinical practice due to the intracranial bleeding from the rupture of these aneurysms, possibly due to the low resolution of DSA or infrequent use of 3D-DSA. Advances in neuroradiological techniques have significantly improved the detection rate of very small aneurysms. In particular, 3D neuroradiological imaging of aneurysms is able to improve the safety of surgery for the treatment of very small aneurysms. However, the surgical treatment of very small aneurysms with direct clipping and endovascular embolization presents considerable difficulty. For example, during the clipping of very small aneurysms (1), the aneurysmal neck may easily break, since very small aneurysms have a thin wall and parent arteries typically exhibit a thick wall with 
calcification. With regard to endovascular embolization of very small aneurysms, maneuvering coils inside the sac of the very small aneurysm is technically difficult, and the walls of the aneurysms can be easily punctured (12). For wide-necked very small aneurysms, despite assistance with stents, coils inside the aneurysms migrate easily, potentially leading to cerebral infarction (1). Brinjikji et al (12) performed a meta-analysis of published studies on endovascular treatment for very small intracranial aneurysms and reported that $\sim 61 \%$ of the aneurysms ruptured and $\sim 39 \%$ did not rupture. The procedure rupture rate for very small aneurysms was $8.3 \%$, and the associated mortality rate was $2.4 \%$. By contrast, the morbidity rate due to thromboembolic complication was $1.9 \%$, and subarachnoid hemorrhage within 1 month post-procedure occurred in $1.6 \%$ of cases. Thus, the meta-analysis indicated that endovascular embolization is feasible and effective for treating $>90 \%$ of very small aneurysms; however, the technique is associated with a high risk of procedure-associated aneurysm rupture (12). Previous studies have indicated that the rupture rate following endovascular embolization of very small aneurysms is $4 \%(10,13)$, which is considerably lower compared with the $8.3 \%$ reported by Brinjikji et al (12). In addition, numerous studies have suggested that endovascular embolization is safe and effective for the treatment of very small aneurysms (13-15). In accordance with these studies, no procedure-associated rupture or thromboembolic complications were observed in the nine patients included in the present study, and the follow-up DSA examinations presented complete occlusion of the aneurysms. Similarly, Zhao et al reported that Solitaire AB stent-assisted coiling embolization effectively treated saccular and dissected intracranial aneurysms, including ruptured and unruptured very small aneurysms (7). Furthermore, Zhou et al hypothesized that coils should remain inside the aneurysms, not in the parent arteries, to prevent hemodynamic alterations in the parent arteries. In addition, the authors proposed that stents should be used to assist coiling to fix coils within the aneurysms, improve the coil packing density in the neck and increase the embolization rate (16).

To date, a number of types of stent have been used in clinical practice, including Neuroform, light-emitting diode, Enterprise and Solitaire stents (3). In addition, numerous stent deployment techniques have been developed for the treatment of aneurysms, including jailing, semi-releasing, stenting after coiling, coil-through, coil-stent, Y-stenting, horizontal-stenting, and single- or multiple-stenting techniques. Stenting after coiling techniques and semi-jailing techniques are the most widely employed in clinical practice $(3,5,17,18)$. The Solitaire AB stent is a self-expanding electric detachable stent with one open end and a closed-mesh design. This design affords specific advantages for the Solitaire AB stent. Firstly, the open-end characteristics facilitate the passing of the stent through tortuous vessels. Secondly, the closed-mesh design increases the radial strength of the stent and its resistance to bending, and makes the stents also favorable for vessels with 2-3 folds overlapping the stent, leading to lower stent porosity $(7,19)$. Thirdly, the Solitaire AB stent is fully retrievable prior to complete deployment. This is the most prominent feature of the Solitaire stent and permits accurate deployment or redeployment of the stent during the procedure $(7,19)$.
In the present study, coil migration into the parent artery following the complete release of the stent was observed in a single case. As the pore in the stent was larger than the diameter of the coil, the coil and stent were retrieved, and subsequently redeployed and released, resulting in stabilization of the coil. Stabilization of the coil following redeployment may be due to the increased stent porosity following retrieval or the positioning of the stent in the aneurysmal neck to block coil movement. Since Solitaire AB stents are fully retrievable, for all patients in the present study, a semi-releasing method was adopted, which allowed for the adjustment of the position of the microcatheter as required during surgery. Thus, based on the coil-filling condition, the time at which the microcatheter was able to be retrieved and the length of the coil outside the aneurysm were determinable. In this manner, the length of the coil may be adjusted to avoid aneurysm rupture due to excessive tension inside the aneurysm and to prevent puncture of the aneurysmal wall by the microcatheter following coil detachment. In addition, the use of Solitaire AB stents permits compression of the coil within the aneurysms, particularly in the aneurysmal neck, which leads to an increased coil packing density in the aneurysm. In the present study, no aneurysm-associated hemorrhage was observed in any of the nine patients, and of the three patients with Raymond grade II occlusion after the surgery, two patients reached Raymond grade I during the follow-up period.

Filling two or more coils in very small aneurysms is difficult and potentially harmful; thus, based on the understanding of the size and anatomy of the aneurysms from 3D-DSA results, one properly selected coil is hypothesized to be appropriate for very small aneurysms. A 3D spherical coil with a diameter less than or equal to the aneurysmal diameter should be the first choice coil for very small aneurysms. This type of coil can be maintained in an aneurysm with less possibility of migration out of the aneurysm, while reducing the possibility of an aneurysm rupture due to the increased compression on the aneurysmal wall from the coil after release of the stent. If a coil in the program is considered to be inappropriate, the coil should be discarded and replaced immediately with an appropriate coil. In addition, a small section of the coil may be left in the parent artery, which is compressed onto the parent artery wall by the stent, to prevent coil prolapse into the parent artery and subsequent cerebral ischemia. This method may facilitate the embolization of very small aneurysms.

In conclusion, endovascular treatment of very small aneurysms presents considerable difficulties and high risk for neurosurgeons. Preoperative determination of the 3D structure of an aneurysm and intraoperative selection of an appropriate coil are important for the success of the surgery. The Solitaire AB stent may be fully retrieved a number of times, and the stent porosity is adjustable, which promotes the success rate of the stent-assisted coiling embolization technique for the treatment of very small aneurysms. In the present study, Solitaire AB stent-assisted coiling embolization was effective and safe for the treatment of ruptured very small intracranial aneurysms in nine patients. However, the sample size of the present study was limited. Future studies with a larger sample size are required to investigate the reopening of aneurysms and the stenosis of parent arteries, in addition to the long-term safety and efficacy of Solitaire AB 
stent-assisted coiling embolization for the treatment of very small aneurysms.

\section{References}

1. Zhang HQ: Is it necessary to treat the mini unruptured intracranial aneurysms? Chin J Cerebrovasc Dis 10: 1-3, 2013.

2. van Rooij WJ, Sprengers ME, de Gast AN, Peluso JP and Sluzewski M: 3D rotational angiography: The new gold standard in the detection of additional intracranial aneurysms. AJNR Am J Neuroradiol 29: 976-979, 2008.

3. Gross BA and Frerichs KU: Stent usage in the treatment of intracranial aneurysms: Past, present and future. J Neurol Neurosurg Psychiatry 84: 244-253, 2013.

4. Almekhlafi MA, Hockley A, Wong JH and Goyal M: Temporary Solitaire stent neck remodeling in the coiling of ruptured aneurysms. J Neurointerv Surg 5 (Suppl 3): iii76-iii78, 2013.

5. Gory B, Klisch J, Bonafé A, et al: Solitaire AB stent-assisted coiling of wide-necked intracranial aneurysms: Short-term results from a prospective, consecutive, European multicentric study. Neuroradiology 55: 1373-1378, 2013.

6. Clajus C, Sychra V, Strasilla C and Klisch J: Stent-assisted coil embolization of intracranial aneurysms using the Solitaire ${ }^{\mathrm{TM}} \mathrm{AB}$ Neurovascular Remodeling Device: Initial and midterm follow-up results. Neuroradiology 55: 629-638, 2013.

7. Zhao KJ, Zhang YW, Xu Y, et al: Reconstruction of saccular and dissected intracranial aneurysms using Solitaire ${ }^{\mathrm{TM}}$ AB stents. PLoS One 8: e57253, 2013.

8. Roy D, Milot G and Raymond J: Endovascular treatment of unruptured aneurysms. Stroke 32: 1998-2004, 2001.

9. Bonita R and Beaglehole R: Recovery of motor function after stroke. Stroke 19: 1497-1500, 1988.

10. Hong B, Yang PF, Zhao R, et al: Endovascular treatment of ruptured tiny intracranial aneurysms. J Clin Neurosci 18: $655-660,2011$
11. Gupta V, Chugh M, Jha AN, Walia BS and Vaishya S: Coil embolization of very small ( $2 \mathrm{~mm}$ or smaller) berry aneurysms: Feasibility and technical issues. AJNR Am J Neuroradiol 30: 308-314, 2009.

12. Brinjikji W, Lanzino G, Cloft HJ, Rabinstein A and Kallmes DF. Endovascular treatment of very small ( $3 \mathrm{~mm}$ or smaller) intracranial aneurysms: Report of a consecutive series and a meta-analysis. Stroke 41: 116-121, 2010.

13. Lu J, Liu JC, Wang LJ, Qi P and Wang DM: Tiny intracranial aneurysms: Endovascular treatment by coil embolisation or sole stent deployment. Eur J Radiol 81: 1276-1281, 2012.

14. Fang C, Li MH, Zhu YQ, et al: The effectiveness and feasibility of endovascular coil embolization for very small cerebral aneurysms: mid- and long-term follow-up. Ann Vasc Surg 24: 400-407, 2010

15. Im SH, Han MH, Kwon OK, et al: Endovascular coil embolization of 435 small asymptomatic unruptured intracranial aneurysms: Procedural morbidity and patient outcome. AJNR Am J Neuroradiol 30: 79-84, 2009.

16. Zhou C, Hong QH, Zhao R, Xu Y, Hong B and Zhao WY: Stent-assisted coil embolization of ruptured anterior communicating artery tiny aneurysms. Chin J Cerebrovasc Dis 10: 9-12, 2013.

17. Martínez-Galdámez M, Saura P, Saura J, Martínez A, De Campos JM and Pérez A: Y-stent-assisted coil embolization of anterior circulation aneurysms using two Solitaire AB devices: A single center experience. Interv Neuroradiol 18: $158-163,2012$.

18. Sychra V, Klisch J, Werner M, et al: Waffle-cone technique with Solitaire ${ }^{\mathrm{TM}}$ AB remodeling device: Endovascular treatment of highly selected complex cerebral aneurysms. Neuroradiology 53: 961-972, 2011.

19. Klisch J, Clajus C, Sychra V, et al: Coil embolization of anterior circulation aneurysms supported by the Solitaire AB Neurovascular Remodeling Device. Neuroradiology 52: 349-359, 2010. 